\title{
Tumor de Krukenberg del ovario asociado a teratoma maduro
}

\author{
Francisco Mucientes $H^{1}{ }^{1}$, Pablo Mucientes $B^{1}{ }^{1}$, Fernando Heredia M. $^{2}$, Susan Ojeda M. ${ }^{2}$, \\ Juan Villanueva G. ${ }^{3}$, Lionel Correa S. ${ }^{3}$, Flor Vergara S. ${ }^{3}$
}

Sección Patología ${ }^{1}$, Departamento Ginecología ${ }^{2}$, Facultad de Medicina, Universidad de Concepción. Servicio de Ginecología ${ }^{3}$, Hospital Clínico Regional de Concepción. Concepción, Chile.

\section{RESUMEN}

Se reporta un caso excepcional de tumor de Krukenberg ovárico bilateral asociado a teratoma maduro encontrado en una mujer de 54 años. La ecografía mostró al lado derecho tumor ovárico sólido de $55 \mathrm{~mm}$ y al lado izquierdo tumor quístico de $125 \mathrm{~mm}$. Se realizó histerectomía total, salpingooforectomía bilateral, resección del epiplón mayor y muestras peritoneales. Al tercer día postcirugía, la paciente presentó signos de tromboembolismo pulmonar masivo y aunque recibió terapia anticoagulante falleció al quinto día postoperatorio. El estudio histológico mostró infiltración masiva de carcinoma de células en anillo positivas para citoqueratina en ambos ovarios. El ovario derecho mostró la forma sólida clásica del tumor de Krukenberg mientras que el ovario izquierdo correspondió a un quiste dermoide con infiltración tumoral de carcinoma de células en anillo en la pared.

\section{PALABRAS CLAVE: Tumor de Krukenberg, teratoma maduro, quiste dermoides, metástasis ováricas, metástasis tumor a tumor}

\section{SUMMARY}

An exceptional case of bilateral Krukenberg tumor of the ovary associated with mature teratoma presented in a 54 years old patient is reported. The ultrasound showed a $55 \mathrm{~mm}$ solid right ovarian tumor and a $125 \mathrm{~mm}$ left cystic ovarian tumor. Hysterectomy and bilateral salpingoophorectomy was performed including omental resection and peritoneal biopsies. Massive pulmonary embolism was detected in the third day after the surgery. Even anticoagulant therapy was established the patient died in the fifth postoperative day. The histological study revealed massive infiltration of signet ring cell carcinoma with positive expression for cytokeratin in both ovaries. The right ovary showed the classical solid form of the tumor. The left ovary was a dermoid cyst with signet ring cell carcinoma infiltrating the cystic wall.

\section{KEY WORDS: Krukenberg tumor, mature teratoma, dermoid cyst, ovarian metastases, tumor to tumor metastasis}

\section{INTRODUCCIÓN}

El ovario frecuentemente recibe metástasis de diversos carcinomas originados en órganos como estómago, colon, mama, apéndice cecal y vesícula biliar entre otros (1). La metástasis ovárica tiene rangos de incidencia variables con una media estimada de $10 \%$ de los tumores ováricos malignos y puede ser la primera manifestación de un carcinoma oculto localizado en un órgano distante $(2,3)$.

El tumor de Krukenberg, en sentido más restrictivo, representa una metástasis ovárica de carcinoma 
de células en anillo de sello (arbitrariamente más del $10 \%$ del tumor) y en la mayoría de los casos originado en el estómago $(3,4)$. La mayor proporción de estos tumores son bilaterales (80\%), tamaño medio de $10 \mathrm{~cm}$, son sólidos y multinodulares, con degeneración quística ocasional y superficie serosa lisa (2).

Excepcionalmente el tumor de Krukenberg puede presentarse concomitantemente con un tumor ovárico de otra estirpe, entre los que se han descrito fibromas (3), tumor de Brenner y cistoadenoma (5) y quistes dermoides $(6,7)$. Esta última asociación solo ha sido mencionada en una serie de tumores de Krukenberg, sin embargo, no hay casos publicados en detalle, lo que motiva nuestro reporte.

\section{Caso clínico}

Paciente de 54 años, con historia de colecistectomía hace 30 años y hernioplastía hace un año, consultó por dolor lumbar y abdominal de un mes de evolución. La ecografía transvaginal mostró útero sin alteraciones, ovario derecho de $55 \times 28$ x $32 \mathrm{~mm}$ sólido homogéneo, volumen de $25,8 \mathrm{ml}$, aspecto tumoral (Figura1), el ovario izquierdo presentaba una lesión quística de $125 \times 116 \times 83 \mathrm{~mm}$, con volumen de $630 \mathrm{ml}$, con septos finos en sus- pensión sugerentes de pelos, y un área central hiperecogénica. Estos hallazgos y el estudio Doppler color con flujo ausente sugirieron el diagnóstico de teratoma (Figura 2). Se realizó histerectomía total, salpingooforectomía bilateral, resección del epiplón mayor, estudio de muestras peritoneales y citológico de lavado peritoneal. Al tercer día presenta cuadro clínico compatible con trombo-embolismo pulmonar masivo del cual fallece al quinto día del postoperatorio. No se realizó autopsia.

Histopatología: El anexo derecho reveló trompa normal y ovario sólido multinodular tumoral de 5,8 $\mathrm{cm}$ (Figura 3). El anexo izquierdo mostró trompa uterina sin alteraciones y ovario quístico de 10,5 $\mathrm{cm}$ de diámetro, biloculado, con grasa, pelos y pequeños nódulos blanquecinos sólidos en la pared (Figura 4). El útero de $62,7 \mathrm{~g}$ y de $8,3 \times 6 \times 3,8$ $\mathrm{cm}$ no mostró alteraciones. Epiplón, muestras peritoneales y citológico peritoneal fueron negativos para neoplasia. La microscopía mostró carcinoma de células en anillo de sello con respuesta estromal fibrosa en ambos ovarios (Figura 5), células que fueron positivas a la inmunotinción para citoqueratina. Además en el ovario izquierdo se observó piel y pelos en la cara interna (Figura 6).

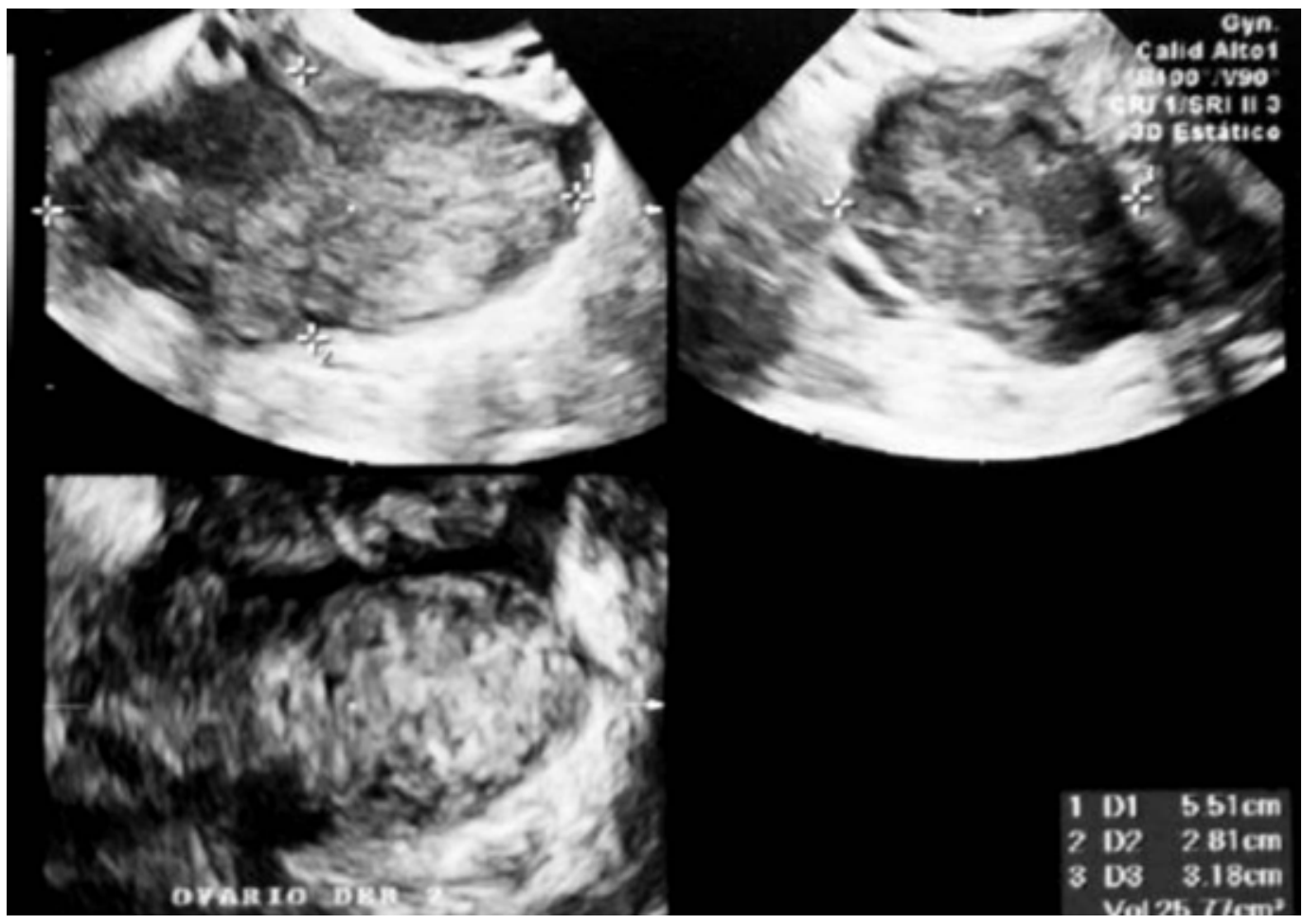

Figura 1. Ecografía transvaginal de tumor sólido del ovario derecho. 


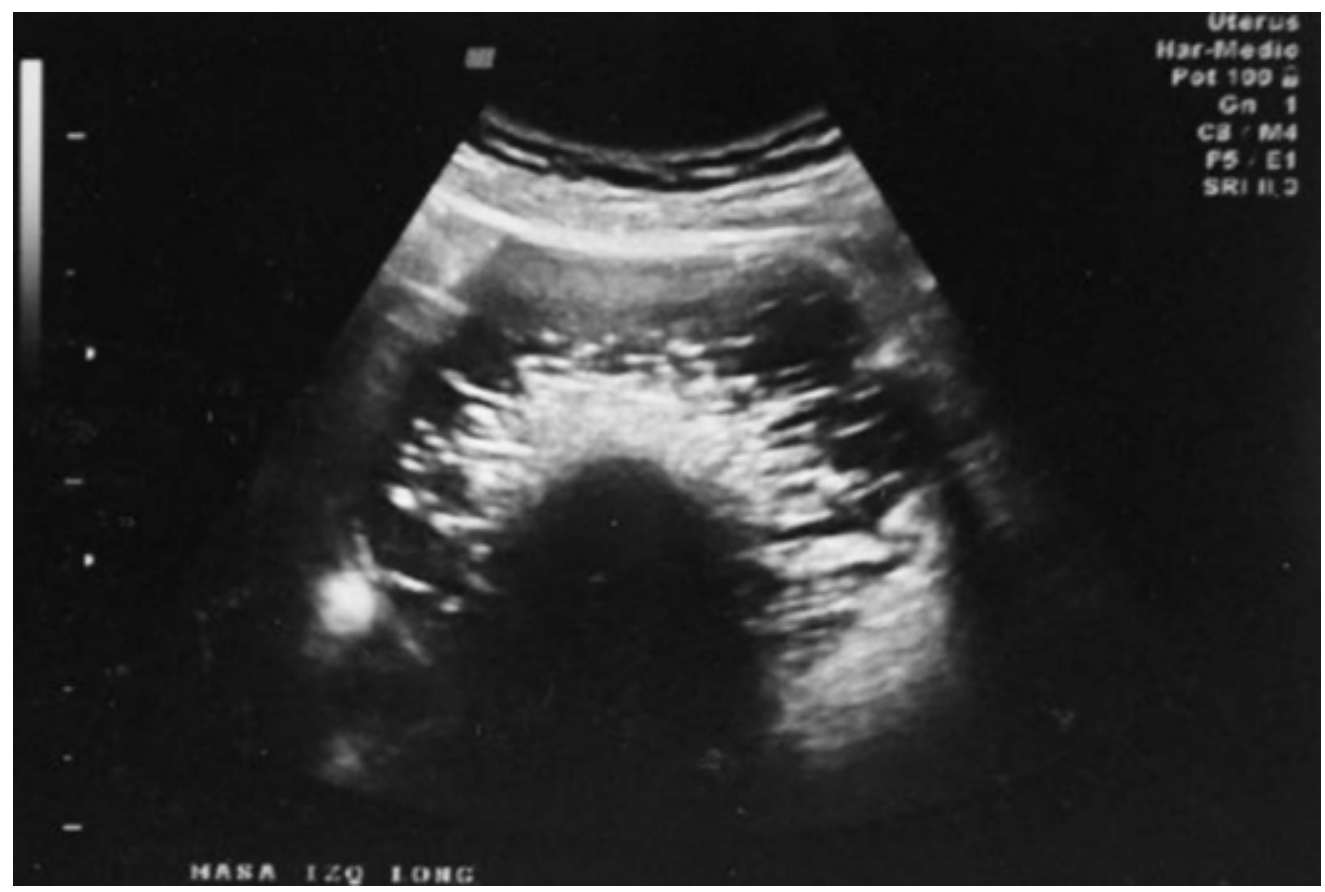

Figura 2. Ecografía transvaginal de tumor quístico del ovario izquierdo.



Figura 3. Vista macroscópica de tumor sólido ovario derecho. 


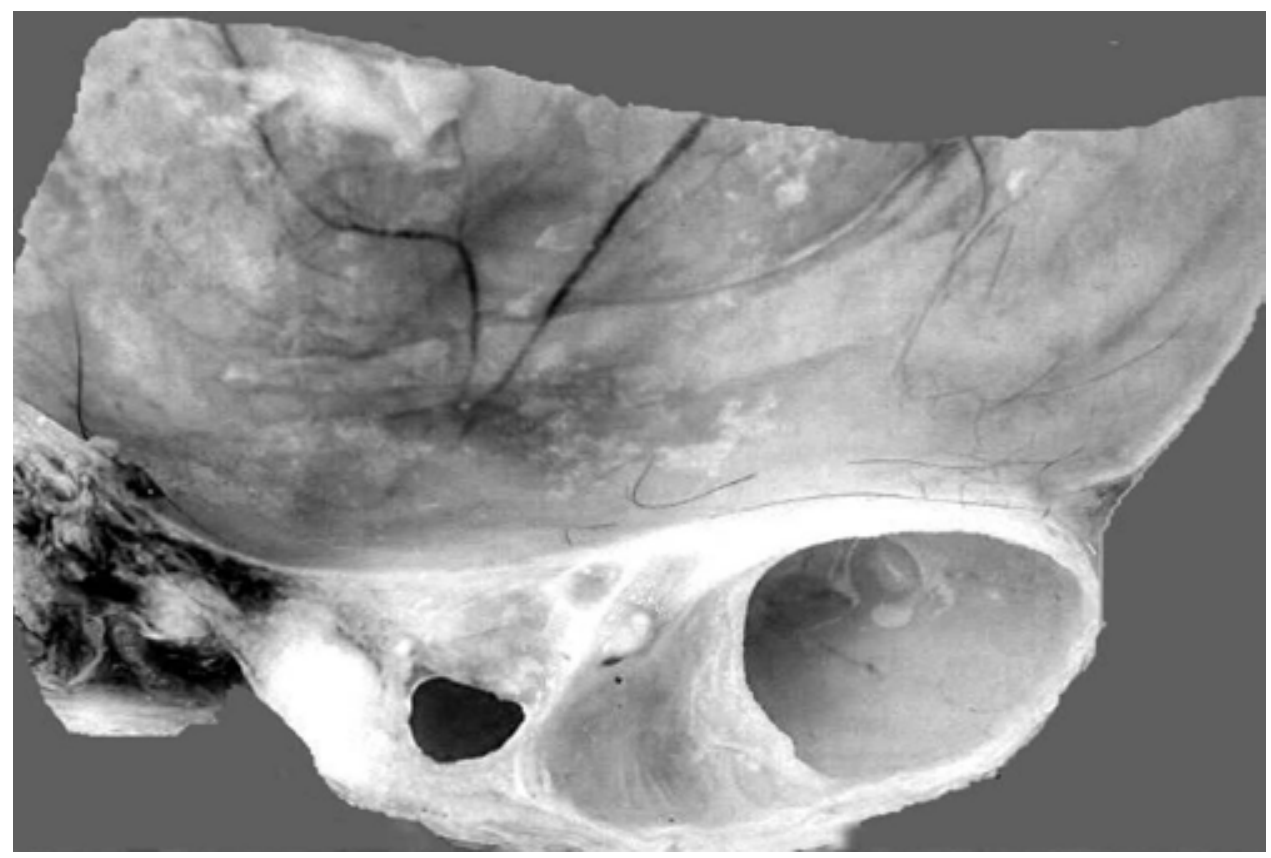

Figura 4. Vista macroscópica del teratoma quístico del ovario izquierdo con nódulos de tumor de Krukenberg en la pared.

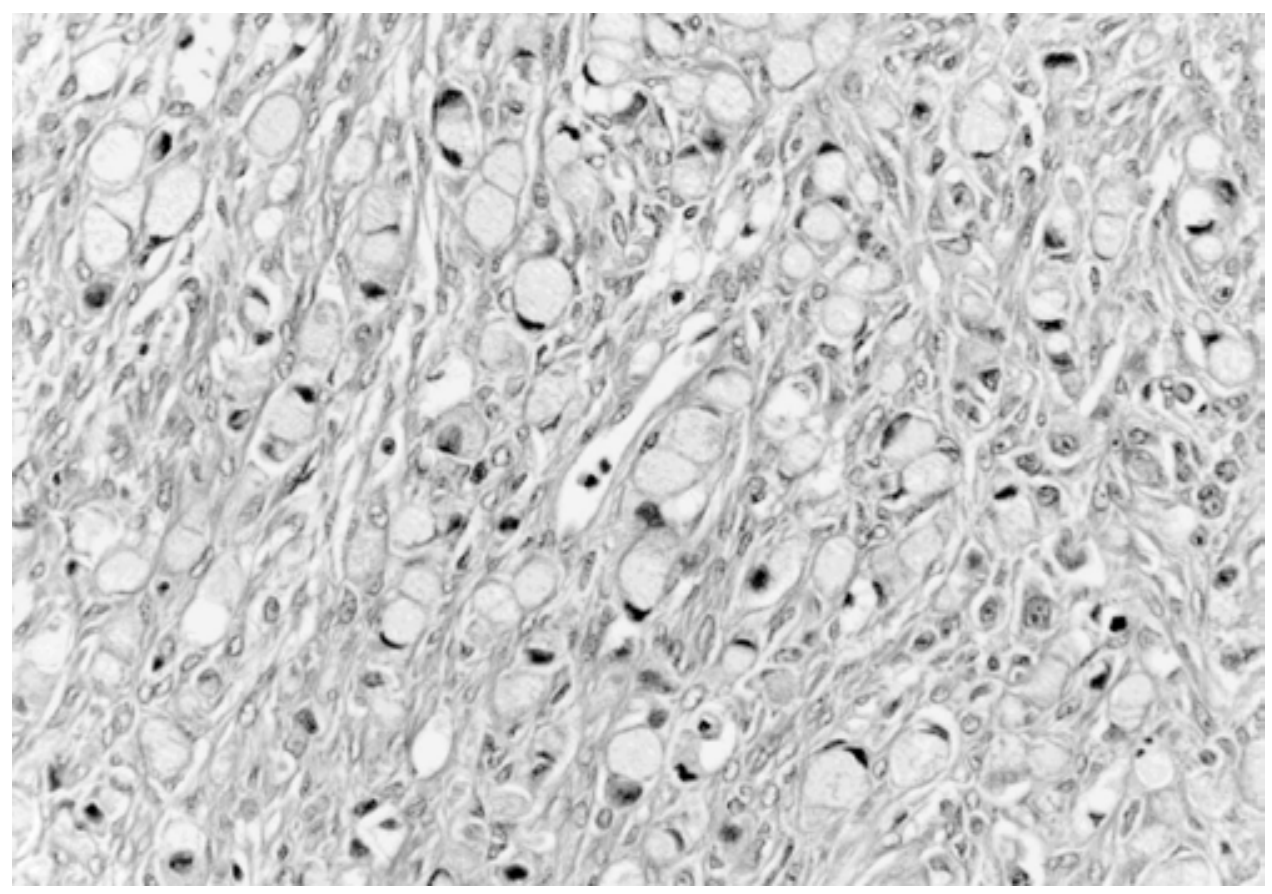

Figura 5. Carcinoma de células en anillo de sello infiltrando la pared del teratoma ovárico izquierdo. 


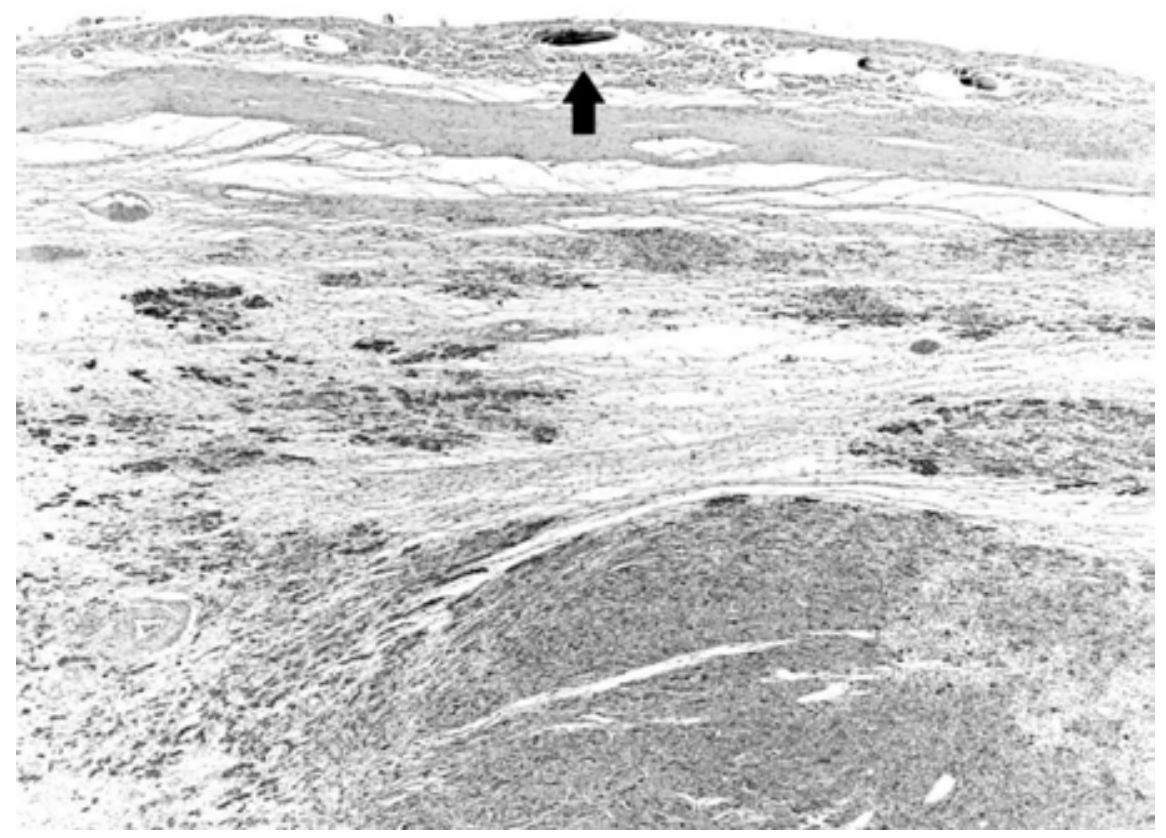

Figura 6. Carcinoma de células en anillo infiltrando la pared del teratoma ovárico izquierdo, positivas a la inmunotinción para queratina, que es también positiva en los pelos en la cara interna (flecha).

\section{DISCUSIÓN}

La ultrasonografía transabdominal y transvaginal se aplican como primera línea de estudio para la evaluación de una masa pélvica (8) aunque con limitaciones por lo que suele complementarse con tomografía computada y resonancia nuclear magnética (9). La ultrasonografía es menos costosa que otros métodos y con un rendimiento diagnóstico similar e incluso mejor para caracterizar cierto tipo de lesiones anexiales. La sensibilidad y especificidad de esta técnica para el diagnóstico de cáncer de ovario alcanza valores de un 86 y $91 \%$ respectivamente al combinar aspectos morfológicos y Doppler (10). Muchos tumores ováricos tienen una apariencia ecográfica típica y altamente predictiva.

Los teratomas suelen tener un nódulo hiperecogénico con sombra acústica dentro de la masa anexial, o presentarse con un relleno característicamente uniforme, a veces como una masa hiperecogénica o con ecos lineales brillantes $(11,12)$. Por otra parte, los principales hallazgos que sugieren malignidad en una masa anexial son un componente sólido no hiperecogénico, a menudo nodular o papilar, presencia de tabiques gruesos, demostración de flujo en el componente sólido, ascitis y eventualmente la detección de masas peritoneales o ganglios linfáticos de mayor tamaño (13). Recientemente se ha usado ultrasonografía con contraste aumentado (CE-US) en el diagnóstico de un tumor de Krukenberg de origen gástrico, permitiendo definir la microvascularización con un refuerzo arterial seguido de una fase de lavado venoso, hallazgos considerados fuertemente sospechosos de malignidad, proponiendo que esta técnica tendría su valor ante la sospecha metástasis ovárica (9).

El cáncer es factor de riesgo de trombo-embolismo venoso incluyendo aumento de morbilidad y mortalidad en adultos y niños $(14,15)$. La frecuencia es mayor en tumores sólidos aunque malignidades hematológicas también contribuyen. A la clásica triada de Virchows: daño endotelial, ectasia y alteraciones de la coagulabilidad, en pacientes con cáncer hay que sumar otra triada recientemente propuesta: biología tumoral, activación de la coagulación y la inflamación (16). Aunque clásicamente se propone prevención con terapia anticoagulante (16), recientemente se ha puesto en duda el valor preventivo de la tromboprofilaxis en pacientes hospitalizados con cáncer (17).

La metástasis desde un tumor a otro tumor está descrita con mayor frecuencia desde carcinomas mamarios y pulmonares a meningiomas (18). Cualquier tumor benigno o maligno puede ser receptor 
de metástasis. Este hecho pudiera ser un evento casual pero hay teorías que involucran vascularización, índice de proliferación, contenido lipídico y moléculas de adhesión celular que proporcionan un ambiente favorable para el desarrollo tumoral (18).

Aunque hay casos bien documentados de tumor de Krukenberg asociado a fibroma ovárico (3), la respuesta hipercelular del estroma ovárico frente a las células carcinomatosas y que en muchos casos es hormonalmente funcional, puede inducir confusión a la histología y ser considerada como el compromiso de un fibroma preexistente, lo que explica el alto número de casos descritos de esta asociación en las primeras series publicadas (4). El hallazgo de quiste dermoide es excepcional y solo se señalan dos casos dentro de una serie de 112 tumores de Krukenberg (7) pero en ninguno de ellos existe un reporte detallado de los hallazgos clínico patológicos. Esta rara asociación puede explicarse en parte por lo poco frecuente del tumor de Krukenberg y por la edad de presentación del teratoma ovárico (media 32 años), un poco menor que la del tumor de Krukenberg (media 45 años) $(3,4)$.

Determinar que un carcinoma en el ovario corresponde a una metástasis o a un tumor primario continúa siendo un desafío diagnóstico. Este hecho es especialmente válido para carcinomas mucinosos, donde un $63 \%$ de estos tumores inicialmente considerados primarios fueron reclasificados como metástasis (19).

Desde el punto de vista práctico, el antecedente de enfermedad tumoral previa, la bilateralidad, el tamaño menor a 10-12 cm, el compromiso seroso, el aspecto multinodular sólido permiten sospechar metástasis en la macroscopía y en la biopsia rápida $(20,21)$. Microscópicamente las embolias tumorales, el compromiso del hilio ovárico, la presencia de muconódulos y la invasión infiltrante apoyan el diagnóstico de metástasis (22). Sin embargo estos criterios tienen limitaciones especialmente en casos de carcinoma mucinoso, limitaciones que se extienden a la inmunohistoquímica que debe incluir un panel de marcadores que pueden no ser del todo específicos para un diagnóstico seguro $(4,22,23,24)$.

Por lo tanto, solo con una evaluación multidisciplinaria se logrará un diagnóstico clínico-patológico confiable. Es necesario reconocer las limitaciones que tiene este desafío diagnóstico y evitar errores al considerar una metástasis ovárica como un carcinoma primario, lo cual impediría efectuar un tratamiento satisfactorio (25)

La mayoría de los autores no acepta que exista un tumor de Krukenberg primario del ovario. Este término se había propuesto para casos de sobrevida larga luego del diagnóstico sin hallazgo de un carcinoma primario o para casos en que una autop- sia completa no reveló un tumor primario extraovárico (4). Sin embargo en este sentido se debe tener en cuenta dos hechos: algunos carcinomas mamarios y gástricos pueden ser muy pequeños para ser clínicamente detectados o pueden permanecer silentes por mucho tiempo, o por otro lado que existen tumores ováricos primarios con células en anillo que tienen un comportamiento biológico diferente y que pueden ser confundidos con una metástasis de carcinoma de células en anillo como son el tumor esclerosante estromal, el tumor estromal de células en anillo, el carcinoide mucinoso y del tumor de células de Sertoli-Leydig, por lo que se requiere un cuidadoso diagnóstico diferencial histológico apoyado por técnicas de inmunohistoquímica (1).

El hallazgo del tumor primario suele ocurrir en forma subsecuente, usualmente a los seis meses del debut ovárico, sin embargo en muchos casos el tumor primario simplemente no se encuentra (4) o como ocurre con el origen gástrico o mamario el antecedente solo se obtiene en un $20-30 \%$ de los casos (26), lo que implica realizar un estudio clínico exhaustivo en busca del primario, lo que debido al curso clínico de la paciente no fue posible hacer en nuestro caso.

El tumor de Krukenberg tiene alta mortalidad con una sobrevida media de 14 meses (4) y peor aun cuando su origen es gástrico (27). Sin embargo se ha propuesto que la cirugía del tumor ovárico podría tener algún beneficio en la sobrevida en pacientes con cáncer gástrico (28), además de evitar la posible complicación de rotura o torsión frecuente en tumores pelvianos libres de gran tamaño.

\section{CONCLUSIÓN}

La metástasis ovárica es relativamente frecuente y debe ser considerada en el diagnóstico diferencial de tumores sólidos o quísticos ováricos usualmente bilaterales, especialmente en mujeres relativamente jóvenes como suele ocurrir con los de origen gástrico. La forma más restrictiva, representada por el tumor de Krukenberg y que muestra infiltrado de carcinoma de células en anillo de sello, puede comprometer el ovario nativo o excepcionalmente un tumor ovárico coexistente como es el caso presentado asociado a un teratoma maduro. Establecer el diagnóstico representa un desafío que debe involucrar un estudio multidisciplinario completo, y en la biopsia se debe tener en cuenta variados tumores que pueden simularlo, lo que requiere uso de técnicas complementarias de inmunohistoquímica. Lograr un diagnóstico seguro tiene importantes implicancias terapéuticas y de pronóstico para el manejo de estas pacientes. 


\section{REFERENCIAS}

1. McCluggage WG, Wilkinson N. Metastatic neoplasm involving de ovary: a review with emphasis on morphological and immune histochemical features. Histopathology 2005;47:231-47.

2. Young RH. From Krukenberg to today: The ever present problems posed by metastatic tumors in the ovary. Part I. Historical perspective, general principles, mucinous tumors including the Krukenberg tumor. Adv Anat Pathol 2006;13:205-27.

3. Kiyokawa T, Young RH, Scully RE. Krukenberg tumors of the ovary. Am J Surg Pathol 2006;30:277-99.

4. Al-Agha OM, Nicastri AD. An in-depth look at Krukenberg tumor: An overview. Arch Pathol Lab Med 2006;130:1725-30.

5. Flanagan CJ, Race GJ. Bilateral Brenner and Krukenberg tumors with ovarian cystadenomas. AMA Arch Pathol 1954;58:588-91.

6. Tazaki $\mathrm{T}$, Nisshimura $\mathrm{H}$, Ushijima $\mathrm{H}$ et al. Two cases of Krukenberg tumors associated with ovarian dermoid cyst. Igaku Kenkyu 1985;55:69-75.

7. Yakushiji M,Tazaki T, Nishumura $\mathrm{H}$, et al. Krukenberg tumors of the ovary: a clinicopathological analysis of 112 cases. Nihon Sanka Fujinka Gakkai Zasshi 1987;39:479-85.

8. Liu J, Xu Y, Wang J. Ultrasonography, computed tomography and magnetic resonance imaging for diagnosis of ovarian carcinoma. Eur J Radiol 2007;62:32834.

9. Tombesi P, DiVece F, Ermili F, Fabbian F, Sartori S. Role of ultrasonography and contrast-enhanced ultrasonography in a case of Krukenberg tumor. World $\mathrm{J}$ Radiol 2013;5:321-4.

10. Myers ER, Bastian LA, Havrilesky LJ, et al. Management of Adnexal Mass. Evidence Report/Technology Assessment No.130 (Prepared by the Duke Evidence-based Practice Center under Contract No. 29002-0025). AHRQ Publication No. 06-E004, Agency for Healthcare Research and Quality, Rockville, MD February 2006.

11. Jermy $\mathrm{K}$, Luise $\mathrm{C}$, Bourne $\mathrm{T}$. The characterization of common ovarian cysts in premenopausal women. UItrasound Obstet Gynecol 2001;17:140-4.

12. Patel MD, Feldstein VA, Lipson SD, et al. Cystic teratomas of the ovary: diagnostic value of sonography. Am J Roentgenol 1998;171:1061-5.

13. Brown DL, Doubilet PM, Miller FH, et al. Benign and malignant ovarian masses: selection of the most discriminating gray-scale and Doppler sonographic features. Radiology 1998;208:103-110.
14. Khorana AA, Francis CW, Culakova E, et al. Thromboembolism is a leading cause of death in cancer patients receiving outpatient chemotherapy. J Thromb Haemost 2007;5:632-4.

15. Walker AJ, Grainge MJ, Card TR, et al. Venous thromboembolism in children. A population-based cohort study. Thrombosis Research 2014;133:340-4.

16. Piazza G. Venous thromboembolism and cancer. Circulation 2013;128:2614-8.

17. Carrier M, Khorana AA, Moretto $P$, et al. Lack of evidence to support thromboprophylaxis in hospitalized medical patient with cancer. A J Med 2014;127:82-6.

18. Moody P, Murtagh K,Piduru S, et al. Tumor-to-tumor metastasis: pathology and neuro imaging considerations. Int J Clin Exp Pathol 2012;5:367-73.

19. Zaino RJ, Brady MF, Lele SM, et al. Advanced stage mucinous adenocarcinoma of the ovary is both rare and highly lethal. Cancer 2011;117:554-62.

20. Seidman JD, Kurman RJ, Ronnett BM. Primary and metastatic mucinous adenocarcinomas in the ovaries. Incidence in routine practice with a new approach to intraoperative diagnosis. Am J Surg Pathol 2003;27:985-93.

21. Stewart CJR, Brennan BA, Hammond IG, et al. Accuracy of frozen section in distinguishing primary ovarian neoplasia from tumors metastatic to the ovary. Int J Gynecol Pathol 2005;24:356-62.

22. Pinto PB, Derchain SF, Andrade LA. Metastatic mucinous carcinomas in the ovary: a practical approach to diagnosis related to gross aspects and to immunohistochemical evaluation. Int J GynecolPathol 2012;31:3138.

23. Baker PM, Oliva E. Immuno histochemistry as a tool in the differential diagnosis of ovarian tumors: an update. Int J Gynecol Pathol 2005;24:39-55.

24. Yemelvanova AV, Vang R, Judson $\mathrm{K}$, et al. Distinction of primary and metastatic mucinous tumors involving the ovary: analysis of size and laterality data by primary site with reevaluation of an algorithm for tumor classification. Am J Surg Pathol 2008;32:128-38.

25. Soslow RA. Mucinous ovarian carcinoma: slippery business. Cancer 2011;117:451-3.

26. Holtz F, Hart WR. Krukenberg tumors of the ovary: a clinic pathological analysis of 27 cases. Cancer 1982;50:2438-47.

27. McGill FM, Ritter DB, Rickard CS,Kaleya RN. Krukenberg tumors: can management be improved? Gynecol Obstet Invest 1999;48:61-5.

28. Lu LC, Shao YY, Hsu CH, et al. Metastasectomy of Krukenberg tumors may be associated with survival benefits in patients with metastatic gastric cancer. Anticancer Res 2012;32:3397-401. 